\title{
The use of fulvestrant before chemotherapy improves survival in hormone-positive breast cancer: a real-life study
}

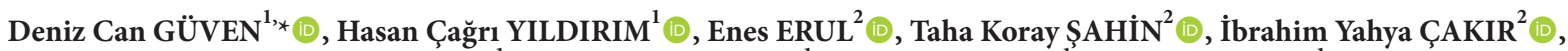 \\ Oktay Halit AKTEPE ${ }^{1}$ (D), Neyran KERTMEN $^{1}$ (D), Ömer DİZDAR ${ }^{1}$ (D), Sercan AKSOY ${ }^{1}$ \\ ${ }^{1}$ Department of Medical Oncology, Hacettepe University Cancer Institute, Ankara, Turkey \\ ${ }^{2}$ Department of Internal Medicine, Hacettepe University Faculty of Medicine, Ankara, Turkey
}

Received: 10.03 .2021

- Accepted/Published Online: 26.08.2021

- Final Version: 19.10 .2022

Background/aim: We aimed to evaluate the efficacy of fulvestrant and its affecting clinical factors, including the optimal sequencing of fulvestrant and chemotherapy in a real-life cohort.

Methods: The data of 256 metastatic hormone-positive breast cancer patients treated with fulvestrant were evaluated. The association of clinical factors with survival was analyzed with Kaplan-Meier and Cox-regression analyses.

Results: The median age of patients was 57 years. More than half of the patients used fulvestrant in later lines and after chemotherapy (75.8\%). The median progression-free (PFS) and overall survival (OS) of all cohort were $6.05 \pm 0.56$ and $29.70 \pm 1.61$ months, respectively. Primary endocrine resistance (HR: 1.989, 95\% CI: 1.430-2.766, <0.001), use of fulvestrant after chemotherapy (HR: 1.849, 95\% CI: $1.182-2.891, \mathrm{p}=0.007$ ) and visceral metastases (HR: 1.587, 95\% CI: $1.128-2.233, \mathrm{p}=0.008$ ) were associated with decreased OS in multivariate analyses. Sixteen patients were treated with trastuzumab and fulvestrant combination. The overall response rate $(\mathrm{p}=0.340)$, disease control rate $(\mathrm{p}=0.076)$, and OS $(\mathrm{p}=0.289)$ and PFS $(\mathrm{p}=0.276)$ were similar to overall cohort.

Conclusion: In our experience, fulvestrant treatment was associated with comparable OS to clinical trials in a large cohort of patients. Patients treated with fulvestrant before chemotherapy were garnered significantly more benefit.

Key words: Fulvestrant, hormone-positive, breast cancer, real-life, FALCON study

\section{Introduction}

Breast cancer is a global health problem and the most important cause of cancer mortality in women worldwide [1]. Significant improvements have been made in the last 20 years in metastatic breast cancer with the advent of targeted therapies in HER-2 positive disease and endocrine treatments in hormone-positive disease [2,3]. Fulvestrant and cyclin-dependent kinase (CDK) inhibitors were added to treatment armamentarium in addition to tamoxifen and aromatase inhibitors in metastatic hormone-positive breast cancer in the last decade and are widely used in clinical practice [4].

Fulvestrant is an estrogen receptor antagonist that blocks estrogen receptor dimerization and DNA binding with the presumed elimination of tamoxifen's agonist effects [5]. The phase III FALCON demonstrated the superiority of fulvestrant over aromatase inhibitors. In this study, aromatase inhibitor and fulvestrant treatments were compared in patients with hormone-positive metastatic breast cancer who had not previously received endocrine therapy. Progression-free survival was 16.6 months in the fulvestrant arm and 13.8 months in the aromatase inhibitor arm, and fulvestrant became one of the standards of care options in the

first-line of treatment of hormone-positive metastatic breast cancer [6].

Although fulvestrant has gained an important place in treating metastatic hormone-positive breast cancer and is widely used in clinical practice, real-life data is scarce. In addition, fulvestrant is often used after chemotherapy in daily practice, but data on this practice is also very limited. In a multicenter study from Italy, the data of 490 breast cancer patients treated with fulvestrant were evaluated and reported an overall survival of 26.8 months. However, a high frequency of endocrine sensitivity (80\%) and use of fulvestrant as maintenance after chemotherapy in a significant percentage of patients hardened the generalizations of the results, especially to limited-

\footnotetext{
^Correspondence: denizcguven@hotmail.com
} 
resource settings [7]. From these points, we aimed to evaluate the efficacy of fulvestrant and affecting clinical factors, including the optimal sequencing of fulvestrant and chemotherapy in a real-life setting.

\section{Methods}

\subsection{Patients}

The medical records of metastatic hormone-receptorpositive breast cancer patients treated with fulvestrant between 09.2005 and 01.2020 in Hacettepe University Cancer Center were retrospectively evaluated. All patients treated in the prespecified dates were included in the analyses other than patients treated in the context of clinical trials, patients treated with fulvestrant and other endocrine treatment combinations.

Baseline demographic features (age, sex, menopause status), histologic subtypes, HER-2 expression status, tumor grades, patterns of metastases (visceral, soft tissue, bone), endocrine sensitivity status (primary resistance, secondary resistance, and endocrine sensitive), the use of trastuzumab with fulvestrant and previous lines of treatment before fulvestrant recorded together with the best response under treatment, date, and pattern of progression under fulvestrant, clinical benefit rate [8] and survival data. Endocrine sensitivity was categorized according to the definitions in the 5th ESO-ESMO international consensus guidelines for advanced breast cancer (ABC5) guideline[9], and CBR was defined as the best response of complete response, partial response, or stable disease lasting more than 24 weeks as suggested in the previous clinical trials [6],[10].

\subsection{Statistical analyses}

The descriptive features were expressed with medians, standard errors, frequencies and percentages wherever appropriate. Baseline characteristics of the fulvestrant plus trastuzumab cohort and the remaining patients were compared with chi-square and Fischer's exact test. Survival times were reported with medians and standard errors (se). The overall survival (OS) time was defined as the period from treatment initiation to the last follow-up and/or death, and progression-free survival (PFS) time was defined as the period between treatment initiation to disease progression and/or death. Survival analyses were conducted with Kaplan-Meier analyses and comparisons of survival times were between prognostic subgroups were done using the log-rank test. Multivariate survival analyses were conducted by a backward Cox-regression model, including the statistically significant parameters in the univariate analyses, and hazard ratios were calculated together with 95\% confidence intervals (CI). Statistical Package for Social Sciences version 20 program was used in the analyses. P-values below 0.05 were considered statistically significant.

\section{Results}

\subsection{Baseline characteristics}

A total of 256 patients were included in the study. The median age of patients was $56.75 \pm 0.71$, and almost all patients were female (99.2\%). The invasive carcinoma of no special type (NST) was the most common histology $(72.9 \%)$, followed by the invasive lobular carcinoma $(10.8 \%)$ and mixed histologies (10.8\%). Most of the patients had moderate to poorly differentiated tumors (91.5\%). Most of the patients' tumors were positive for ER+ and PR+ while there were a small number of ER+, PR- and ER-, PR+ cases. A small group of patients (6.25\%) had HER-2 positivity defined by a $3+$ IHC or FISH positivity for HER-2. More than half of the patients used fulvestrant in the later lines of treatment (50.7\%) and after chemotherapy (75.8\%). Although only $20.3 \%$ of the patients used fulvestrant as the first-line of treatment, the use of fulvestrant as first-line treatment $(\mathrm{p}<0.001)$ and before chemotherapy ( $\mathrm{p}=0.001)$ was significantly increased after the publishing of the FALCON study [6]. A significant portion of the patients (87.1\%) had some level of endocrine resistance. While the bone-only disease was present in $41.4 \%$ of the patients, $52.4 \%$ of the patients had visceral involvement (Table 1 ).

\subsection{Univariate and multivariate survival analyses}

The median follow-up after the start of fulvestrant was $18.18 \pm 1.20$ months. The overall response rate was $35.9 \%$, and the disease control rate was $53.8 \%$. CBR at the 24 th week was achieved in $46.9 \%$ of the patients. During the follow-up, 211 of 256 patients had progressed or died. The median PFS and OS of all cohort were $6.05 \pm 0.56$ and $29.70 \pm 1.61$ months, respectively. In patients with a partial or complete response to fulvestrant, median OS was more than four years and significantly improved compared to patients without response $(49.61 \pm 1.94$ vs. $24.48 \pm 2.51$, $\mathrm{p}<0.001)$. While the efficacy of fulvestrant was similar in patients with different ages ( $\geq$ vs. $<65, p=0.641$ ), menopausal status $(\mathrm{p}=0.507)$ and histologic subtype $(\mathrm{p}=$ $0.500)$, body mass index $(<25$ or $\geq 25, p=0.614)$; primary endocrine resistance $(\mathrm{p}<0.001)$, use of fulvestrant in later than the first-line $(\mathrm{p}=0.003)$ or after the chemotherapy ( $\mathrm{p}$ $=0.001)$ and presence of visceral metastases $(\mathrm{p}=0.001)$ were associated with a decreased survival with fulvestrant (Figure abc, Table 2). The PFS analyses were consistent with OS analyses other than longer PFS in older patients ( $\geq$ vs. $<65, p=0.017$ ).

Among these factors, primary endocrine resistance, the use of fulvestrant after chemotherapy, and visceral metastases remained significant in the multivariate analyses for OS. The primary endocrine resistance and visceral metastases were also related to decreased PFS in the multivariate analyses (Table 3). 
Table 1. Baseline characteristics of patients.

\begin{tabular}{|c|c|c|c|}
\hline & & Count, $\mathrm{n}$ & Percentage, $\%$ \\
\hline \multirow{2}{*}{ Sex } & Female & 254 & $99.2 \%$ \\
\hline & Male & 2 & $0.8 \%$ \\
\hline \multirow{3}{*}{ Menopausal Status } & Premenopausal & 115 & $45.2 \%$ \\
\hline & Perimenopausal & 11 & $4.3 \%$ \\
\hline & Postmenopausal & 128 & $50.5 \%$ \\
\hline \multirow{2}{*}{ Age } & $<65$ & 198 & $77.3 \%$ \\
\hline & $\geq 65$ & 58 & $22.7 \%$ \\
\hline \multirow{4}{*}{ Histologic Type } & NST & 175 & $72.9 \%$ \\
\hline & ILC & 26 & $10.8 \%$ \\
\hline & Mixed NST plus ILC & 26 & $10.8 \%$ \\
\hline & Other & 13 & $5.4 \%$ \\
\hline \multirow{3}{*}{ Grade } & 1 & 17 & $8.5 \%$ \\
\hline & 2 & 111 & $55.2 \%$ \\
\hline & 3 & 73 & $36.3 \%$ \\
\hline \multirow{2}{*}{ ER positivity } & Positive & 228 & $96.6 \%$ \\
\hline & Negative & 8 & $3.4 \%$ \\
\hline \multirow{2}{*}{ PR positivity } & Positive & 199 & $86.1 \%$ \\
\hline & Negative & 32 & $13.9 \%$ \\
\hline \multirow{3}{*}{ Endocrine Sensitivity } & Endocrine sensitive & 33 & $12.9 \%$ \\
\hline & Primary endocrine resistant & 81 & $31.6 \%$ \\
\hline & Secondary endocrine resistant & 142 & $55.5 \%$ \\
\hline \multirow{5}{*}{ Line of Fulvestrant } & 1 & 52 & $20.3 \%$ \\
\hline & 2 & 75 & $29.3 \%$ \\
\hline & 3 & 46 & $18.0 \%$ \\
\hline & 4 & 44 & $17.2 \%$ \\
\hline & 5 or over & 39 & $15.2 \%$ \\
\hline \multirow{2}{*}{$\begin{array}{l}\text { Chemotherapy before } \\
\text { Fulvestrant }\end{array}$} & Absent & 62 & $24.2 \%$ \\
\hline & Present & 194 & $75.8 \%$ \\
\hline \multirow{2}{*}{ Fulvestrant plus trastuzumab } & Absent & 240 & $93.7 \%$ \\
\hline & Present & 16 & $6.3 \%$ \\
\hline \multirow{4}{*}{ Metastasis sites } & Bone & 106 & $41.4 \%$ \\
\hline & Visceral & 55 & $21.5 \%$ \\
\hline & Bone plus visceral & 79 & $30.9 \%$ \\
\hline & Soft tissue & 16 & $6.3 \%$ \\
\hline \multirow{2}{*}{ Metastasis pattern } & Nonvisceral & 122 & $47.7 \%$ \\
\hline & Visceral & 134 & $52.3 \%$ \\
\hline \multirow{2}{*}{$\begin{array}{l}\text { Timing according to } \\
\text { FALCON study }\end{array}$} & Before FALCON study & 138 & $53.9 \%$ \\
\hline & After FALCON study & 118 & $46.1 \%$ \\
\hline
\end{tabular}

${ }^{\star}$ NST: invasive carcinoma of no special type, ILC: invasive lobular carcinoma, ER: estrogen receptor, PR: progesterone receptor 

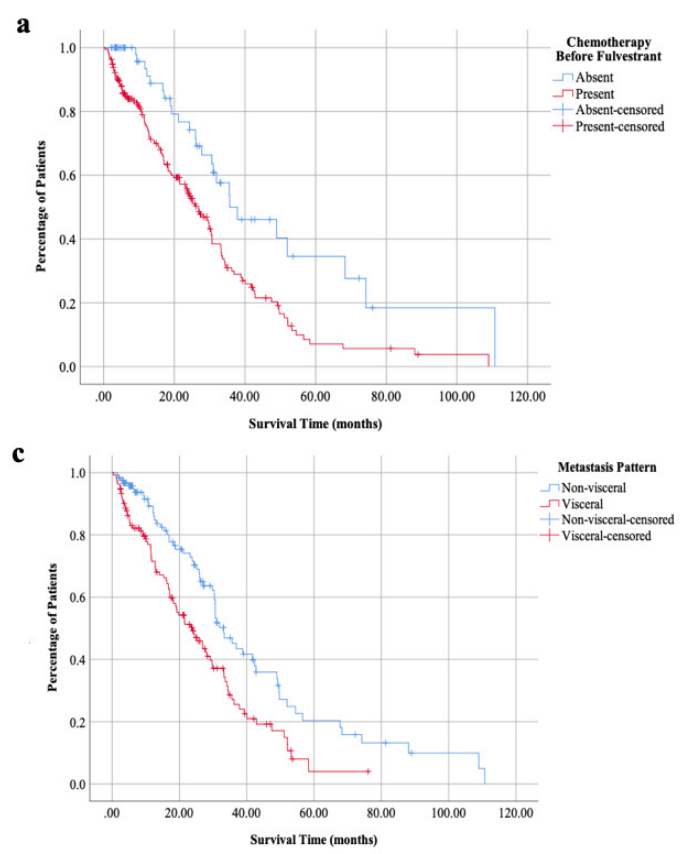

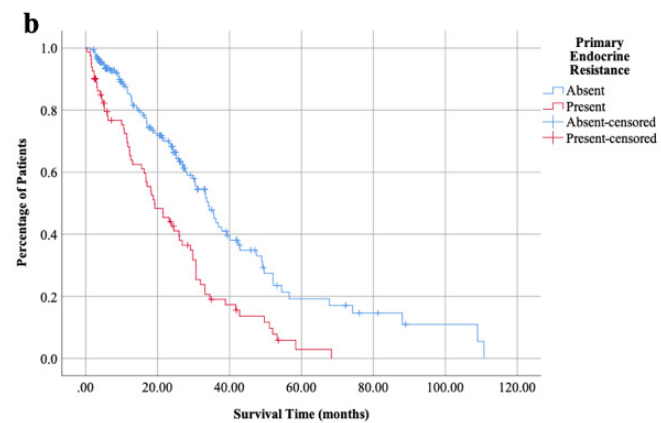

d

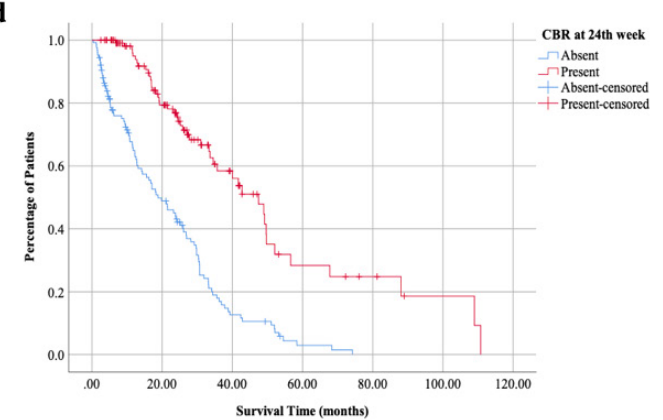

Figure. Overall survival according to chemotherapy before fulvestrant (a), primary endocrine resistance (b), metastasis pattern (c) and clinical benefit rate at the $24^{\text {th }}$ week (d).

Table 2. Median progression-free and overall survival times in univariate analyses.

\begin{tabular}{|l|l|l|l|}
\hline \multicolumn{2}{|c|}{} & PFS (months \pm se) & OS (months \pm se) \\
\hline \multirow{3}{*}{ Age } & $<65$ & $5.52 \pm 0.43$ & $29.70 \pm 1.55$ \\
\cline { 2 - 4 } & $\geq 65$ & $9.53 \pm 1.97$ & $31.01 \pm 6.99$ \\
\hline \multirow{4}{*}{ Menopausal status } & Premenopausal & $5.39 \pm 0.59$ & $28.16 \pm 3.22$ \\
\cline { 2 - 4 } & Perimenopausal & $7.92 \pm 1.63$ & $17.08 \pm 7.45$ \\
\cline { 2 - 4 } BMI & Postmenopausal & $7.13 \pm 1.22$ & $30.36 \pm 1.77$ \\
\hline \multirow{3}{*}{ Endocrine sensitivity } & $<25$ & $5.91 \pm 1.02$ & $29.70 \pm 2.83$ \\
\cline { 2 - 4 } & $\geq 25$ & $6.18 \pm 0.94$ & $29.14 \pm 2.33$ \\
\hline \multirow{2}{*}{ Metastasis Pattern } & Endocrine sensitive & $18.69 \pm 0.78$ & $33.61 \pm 5.39$ \\
\cline { 2 - 4 } & Primary resistance & $3.75 \pm 0.26$ & $19.15 \pm 3.18$ \\
\cline { 2 - 4 } & Secondary resistance & $7.23 \pm 1.02$ & $33.35 \pm 2.51$ \\
\hline \multirow{2}{*}{ Fulvestrant line } & Visceral & $5.13 \pm 0.57$ & $23.89 \pm 3.44$ \\
\cline { 2 - 4 } & Nonvisceral & $8.58 \pm 1.92$ & $33.15 \pm 2.52$ \\
\hline \multirow{2}{*}{ Chemotherapy before fulvestrant } & First-line & $11.30 \pm 1.99$ & $48.95 \pm 8.36$ \\
\cline { 2 - 4 } & After first-line & $5.39 \pm 0.41$ & $26.94 \pm 2.12$ \\
\cline { 2 - 4 } & Present & $9.46 \pm 1.38$ & $26.84 \pm 2.07$ \\
\hline
\end{tabular}

*BMI: body mass index 


\subsection{Fulvestrant plus trastuzumab cohort}

Sixteen patients were treated with a combination of trastuzumab and fulvestrant. The menopausal status, patient age ( $\geq 65$ vs. $<65$ ), tumor grade, metastasis pattern (visceral vs. nonvisceral), endocrine sensitivity, and treatment lines of fulvestrant were similar to the general cohort in patients treated with fulvestrant plus trastuzumab. All patients in this cohort used fulvestrant after chemotherapy. The overall response rate $(\mathrm{p}=0.340)$, disease control rate $(\mathrm{p}=0.076)$, as well as the OS ( $p=0.289)$ and PFS $(\mathrm{p}=0.276)$, were similar to the overall cohort in patients treated with fulvestrant plus trastuzumab combination (Table 4).

Table 3. Multivariate analyses for progression-free and overall survival.

\begin{tabular}{|l|l|l|l|}
\hline Progression-free survival & HR & 95\% confidence interval & P-value \\
\hline Primary endocrine resistance & 2.345 & $1.749-3.145$ & $<0.001$ \\
\hline Fulvestrant after the first-line & 1.280 & $0.872-1.880$ & 0.208 \\
\hline Chemotherapy before fulvestrant & 1.134 & $0.740-1.738$ & 0.564 \\
\hline Visceral metastases presence & 1.361 & $1.030-1.799$ & 0.030 \\
\hline Age $(\geq$ or $<65)$ & 0.716 & $0.502-1.021$ & 0.065 \\
\hline Overall survival & HR & $\mathbf{9 5 \%}$ CI & P-value \\
\hline Primary endocrine resistance & 1.989 & $1.430-2.766$ & $<0.001$ \\
\hline Fulvestrant after the first-line & 1.435 & $0.753-2.735$ & 0.273 \\
\hline Chemotherapy before fulvestrant & 1.849 & $1.182-2.891$ & 0.007 \\
\hline Visceral metastases presence & 1.587 & $1.128-2.233$ & 0.008 \\
\hline
\end{tabular}

Table 4. Comparison of HER-2 positive patients to other patients.

\begin{tabular}{|c|c|c|c|c|}
\hline & & Fulvestrant plus herceptin cohort $(n=16)$ & Other patients $(n=240)$ & P-value \\
\hline \multirow{2}{*}{ Age group } & $\geq 65$ years of age & $2(12.5 \%)$ & $56(23.3 \%)$ & \multirow{2}{*}{0.537} \\
\hline & $<65$ years of age & $14(87.5 \%)$ & $184(76.7 \%)$ & \\
\hline \multirow{3}{*}{ Grade } & 1 & 0 & $17(9 \%)$ & \multirow{3}{*}{0.201} \\
\hline & 2 & $5(41.7 \%)$ & $106(56 \%)$ & \\
\hline & 3 & $7(58.3 \%)$ & $66(35 \%)$ & \\
\hline \multirow{3}{*}{$\begin{array}{l}\text { Menopause } \\
\text { status }\end{array}$} & Premenopausal & $107(45 \%)$ & $8(50 \%)$ & \multirow{3}{*}{0.827} \\
\hline & Perimenopausal & $10(4.2 \%)$ & $1(6.3 \%)$ & \\
\hline & Postmenopausal & $121(50.8 \%)$ & $7(43.7 \%)$ & \\
\hline \multirow{2}{*}{$\begin{array}{l}\text { Metastasis } \\
\text { pattern }\end{array}$} & Nonvisceral & $6(37.5 \%)$ & $116(48.3 \%)$ & \multirow{2}{*}{0.401} \\
\hline & Visceral & $10(62.5 \%)$ & $124(51.7 \%)$ & \\
\hline \multirow{2}{*}{ Treatment line } & First-line & $4(25 \%)$ & $48(20 \%)$ & \multirow{2}{*}{0.747} \\
\hline & Second-line or later & $12(75 \%)$ & $192(80 \%)$ & \\
\hline \multirow{3}{*}{$\begin{array}{l}\text { Endocrine } \\
\text { sensitivity }\end{array}$} & Endocrine sensitive & $4(25 \%)$ & $29(12.1 \%)$ & \multirow{3}{*}{0.051} \\
\hline & Primary endocrine resistance & $1(6.3 \%)$ & $80(33.3 \%)$ & \\
\hline & Secondary endocrine resistance & $11(68.7 \%)$ & $131(54.6 \%)$ & \\
\hline \multirow{2}{*}{$\begin{array}{l}\text { Overall response } \\
(\mathrm{CR} / \mathrm{PR})\end{array}$} & Present & $4(25 \%)$ & $80(36.7 \%)$ & \multirow{2}{*}{0.340} \\
\hline & Absent & $12(75 \%)$ & $138(63.3 \%)$ & \\
\hline \multirow{2}{*}{ Disease control } & Present & $4(25 \%)$ & $114(52.3 \%)$ & \multirow{2}{*}{0.076} \\
\hline & Absent & $12(75 \%)$ & $104(47.7 \%)$ & \\
\hline \multicolumn{2}{|c|}{ Progression-free survival, months } & $12.75 \pm 2.67$ & $5.95 \pm 0.53$ & 0.271 \\
\hline \multicolumn{2}{|c|}{ Overall-survival, months } & $28.16 \pm 13.93$ & $29.7 \pm 1.81$ & 0.290 \\
\hline
\end{tabular}




\section{Discussion}

In this real-life study, fulvestrant treatment was associated with almost 30 months OS and a significant disease control rate. While the outcomes were improved with the earlier use of fulvestrant, patients with primary endocrine resistance and visceral metastases had significantly worse outcomes.

Fulvestrant is a feasible and cost-effective treatment option in advanced breast cancer [11]. Although a variety of clinical trials in different treatment lines were available, the real-life data is limited. The real-life setting is very different from the clinical trials due to more unfit patients and more patients treated in the later lines due to physician preferences or reimbursement reasons [12]. The most comprehensive real-life data on the fulvestrant use was a recent article from Italy. In the study, 490 patients were prospectively evaluated. The study design differed from our study due to the inclusion of patients using fulvestrant as maintenance after chemotherapy, an indication which is not reimbursed in our country. The median OS was similar to our study (26.8 vs. 29.7 months), while the PFS was longer, possibly due to more patients using fulvestrant in the first-line and patients using fulvestrant for maintenance. The line of treatment and presence of liver metastases was related to PFS, and liver metastases were also related to poorer OS in the study [7]. In another recent study from China, the use of fulvestrant in the later lines was related to a decreased response rate and progression-free survival. On the contrary, visceral metastases did not affect the outcomes, although the low number of cases $(n=60)$ could have confounded the results [13]. Another reallife study on 306 patients treated with fulvestrant in different treatment lines also reported improved PFS in patients without liver metastases and patients receiving fulvestrant in the first-line or before chemotherapy [14]. In our study, the median OS was 48.95 months in patients treated in the first-line. Similarly, an overall survival of more than 40 months was reported in a similar study with the fulvestrant in the first-line [15]. We think that these figures are comparable to the survival times reported in the first-line CDKi combination trials [16] and support "the earlier, the better" notion with fulvestrant and further strengthen its place in the first-line treatment of metastatic breast cancer as an efficient option.

Several other studies tried to detect clinical features of patients benefitting most from the fulvestrant, but many failed to demonstrate specific features [17]. However, a study reported prolonged PFS with lower $(<25)$ BMI in endocrine-resistant patients, although in the endocrinesensitive cohort, the OS was lower in the $\mathrm{BMI}<25$ group making the generalizability and interpretation of the study results difficult. Earlier treatment with fulvestrant was related to improved outcomes in this study, while HER-2 positive patients had decreased OS contrary to our study [18]. The use of fulvestrant as monotherapy rather than the combination with trastuzumab could be the reason for different results compared to our study. Despite the notion of dual-targeting opportunity in patients with HER-2 and hormone-positive breast cancer, the development of endocrine and anti-HER2 combinations was relatively slow, [19] possibly due to resistance to conventional endocrine treatments in the HER-2 positive breast cancer [20]. Previous trials of aromatase inhibitors with lapatinib or trastuzumab consistently showed improved PFS and response rates, although the lack of overall survival improvements signified the inevitable endocrine resistance in this patient group [21-24]. We think that fulvestrant could be a better partner to anti-HER2 agents in these combinations due to increased activity and a different mechanism of action. In a multicenter study on the 102 HER-2 positive patients, fulvestrant demonstrated more than $40 \%$ clinical benefit rate and retained activity even in the heavily pretreated patients. Only 5 of the patients used concurrent trastuzumab in this study. Interestingly, all five patients experienced a stable disease lasting six or more months, which was very promising [25]. Unfortunately, this combination did not progress in the clinical trials after a phase II study closed due to low accrual (NCT00138125). The interest in the endocrine and anti-HER2 combinations was renewed after the development of CDK 4/6 inhibitors. Improved PFS with the combination of abemaciclib plus fulvestrant plus trastuzumab compared to conventional chemotherapy and trastuzumab combination was very promising in phase II monarchHER study [26]. Further development of this combination is eagerly awaited.

Our study has several limitations. First, most of our patients used fulvestrant in later treatment lines or after the chemotherapy. Our study lacks a comparator arm, so the true benefit of fulvestrant compared to other available treatments is hard to be evaluated from our data. Additionally, the retrospective design and the small number of patients in some subgroups make it hard to reach definitive conclusions in these subgroups, although these results could be hypothesis-generating.

\section{Conclusion}

In our experience, fulvestrant treatment was associated with comparable overall survival and disease control rates to clinical trials in a large cohort of patients in a real-life setting. Patients treated with fulvestrant before chemotherapy and in the first-line were garnered significantly more benefit. In addition, we think that fulvestrant plus trastuzumab could be a chemo-free option in HER-2 positive patients with comparable outcomes to HER-2 negative patients treated with fulvestrant. 


\section{References}

1. Bray F, Ferlay J, Soerjomataram I, Siegel RL, Torre LA et al. Global cancer statistics 2018: GLOBOCAN estimates of incidence and mortality worldwide for 36 cancers in 185 countries. CA Cancer J Clin 2018; 68 (6): 394-424. https://doi. $\operatorname{org} / 10.3322 /$ caac. 21492

2. Tong CWS, Wu M, Cho WCS, To KKW. Recent Advances in the Treatment of Breast Cancer. Frontiers in Oncology 2018; 8 (227). https://doi.org/10.3389/fonc.2018.00227

3. Guo F, Kuo Y-f, Shih YCT, Giordano SH, Berenson AB. Trends in breast cancer mortality by stage at diagnosis among young women in the United States. Cancer 2018; 124 (17): 3500-3509. https://doi.org/10.1002/cncr.31638

4. Aggelis V, Johnston SRD. Advances in Endocrine-Based Therapies for Estrogen Receptor-Positive Metastatic Breast Cancer. Drugs 2019; 79 (17): 1849-1866. https://doi. org/10.1007/s40265-019-01208-8

5. Osborne CK, Wakeling A, Nicholson RI. Fulvestrant: an oestrogen receptor antagonist with a novel mechanism of action. British Journal of Cancer 2004; 90 (1): S2-S6. https:// doi.org/10.1038/sj.bjc.6601629

6. Robertson JFR, Bondarenko IM, Trishkina E, Dvorkin M, Panasci L et al. Fulvestrant $500 \mathrm{mg}$ versus anastrozole $1 \mathrm{mg}$ for hormone receptor-positive advanced breast cancer (FALCON): an international, randomised, double-blind, phase 3 trial. The Lancet 2016; 388 (10063): 2997-3005. https://doi.org/10.1016/ S0140-6736(16)32389-3

7. Palumbo R, Sottotetti F, Quaquarini E, Gambaro A, Ferzi A et al. Patterns of treatment and outcome with 500-mg fulvestrant in postmenopausal women with hormone receptorpositive/HER2-negative metastatic breast cancer: a real-life multicenter Italian experience. Therapeutic Advances in Medical Oncology 2019; 11: 1758835919833864. https://doi. org/10.1177/1758835919833864

8. Lau E, McCoy P, Reeves F, Chow K, Clarkson M et al. Detection of ctDNA in plasma of patients with clinically localised prostate cancer is associated with rapid disease progression. Genome Medicine 2020; 12 (1): 72. https://doi.org/10.1186/s13073-020-00770-1

9. Cardoso F, Paluch-Shimon S, Senkus E, Curigliano G, Aapro MS et al. 5th ESO-ESMO international consensus guidelines for advanced breast cancer (ABC 5). Ann Oncol 2020; 31 (12): 1623-1649. https://doi.org/10.1016/j.annonc.2020.09.010

10. Di Leo A, Jerusalem G, Petruzelka L, Torres R, Bondarenko IN et al. Results of the CONFIRM phase III trial comparing fulvestrant $250 \mathrm{mg}$ with fulvestrant $500 \mathrm{mg}$ in postmenopausal women with estrogen receptor-positive advanced breast cancer. J Clin Oncol 2010; 28 (30): 4594-4600. https://doi.org/10.1200/ jco.2010.28.8415

11. Telford C, Bertranou E, Large S, Phelps H, Ekman M et al. Cost-Effectiveness Analysis of Fulvestrant $500 \mathrm{mg}$ in Endocrine Therapy-Naïve Postmenopausal Women with Hormone Receptor-Positive Advanced Breast Cancer in the UK. PharmacoEconomics - Open 2019; 3 (4): 559-570. https:// doi.org/10.1007/s41669-019-0134-3
12. Booth CM, Karim S, Mackillop WJ. Real-world data: towards achieving the achievable in cancer care. Nature Reviews Clinical Oncology 2019; 16 (5): 312-325. https://doi.org/10.1038/ s41571-019-0167-7

13. Huang J, Huang P, Shao X-y, Sun Y, Lei L et al. Efficacy of fulvestrant $500 \mathrm{mg}$ in Chinese postmenopausal women with advanced/recurrent breast cancer and factors associated with prolonged time-to-treatment failure: A retrospective case series. Medicine 2020; 99 (29): e20821. https://doi.org/10.1097/ md.0000000000020821

14. Lei W, Li H, Song G, Zhang R, Ran R et al. Abstract P5-1120: Efficacy observation of 306 patients with fulvestrant $500 \mathrm{mg}$ treatment in hormone-receptor positive HER2 negative metastasis breast cancer, a real world study. Cancer Research 2020; 80 (4 Supplement): P5-11-20-P15-11-20. https://doi. org/10.1158/1538-7445.Sabcs19-p5-11-20

15. Skinner KE, Olufade T, Walker MS, Schwartzberg LS. Realworld effectiveness of fulvestrant monotherapy as first endocrine treatment in patients with metastatic breast cancer. The Breast Journal 2020; 26 (2): 112-119. https://doi. org/10.1111/tbj.13492

16. Schettini F, Giudici F, Giuliano M, Cristofanilli M, Arpino G et al. Overall survival of CDK4/6-inhibitors-based treatments in clinically relevant subgroups of metastatic breast cancer: systematic review and meta-analysis. JNCI: Journal of the National Cancer Institute 2020. https://doi.org/10.1093/jnci/ djaa071

17. Graham J, Pitz M, Gordon V, Grenier D, Amir E et al. Clinical predictors of benefit from fulvestrant in advanced breast cancer: A Meta-analysis of randomized controlled trials. Cancer Treatment Reviews 2016; 45: 1-6. https://doi. org/10.1016/j.ctrv.2016.02.004

18. Pizzuti L, Natoli C, Gamucci T, Mauri M, Sergi D et al. Anthropometric, clinical and molecular determinants of treatment outcomes in postmenopausal, hormone receptor positive metastatic breast cancer patients treated with fulvestrant: Results from a real word setting. Oncotarget 2017; 8 (40): 69025-69037. https://doi.org/10.18632/oncotarget.16982

19. Montemurro F, Di Cosimo S, Arpino G. Human epidermal growth factor receptor 2 (HER2)-positive and hormone receptor-positive breast cancer: new insights into molecular interactions and clinical implications. Annals of Oncology 2013; 24 (11): 2715-2724. https://doi.org/10.1093/annonc/ mdt 287

20. De Laurentiis M, Arpino G, Massarelli E, Ruggiero A, Carlomagno C et al. A meta-analysis on the interaction between HER-2 expression and response to endocrine treatment in advanced breast cancer. Clin Cancer Res 2005; 11 (13): 47414748. https://doi.org/10.1158/1078-0432.Ccr-04-2569

21. Schwartzberg LS, Franco SX, Florance A, O’Rourke L, Maltzman J et al. Lapatinib plus Letrozole as First-Line Therapy for HER-2+ Hormone Receptor-Positive Metastatic Breast Cancer. The Oncologist 2010; 15 (2): 122-129. https:// doi.org/10.1634/theoncologist.2009-0240 


\section{GÜVEN et al. / Turk J Med Sci}

22. Johnston S, Pippen J, Jr., Pivot X, Lichinitser M, Sadeghi S et al. Lapatinib combined with letrozole versus letrozole and placebo as first-line therapy for postmenopausal hormone receptor-positive metastatic breast cancer. J Clin Oncol 2009; 27 (33): 5538-5546. https://doi.org/10.1200/jco.2009.23.3734

23. Huober J, Fasching PA, Barsoum M, Petruzelka L, Wallwiener $D$ et al. Higher efficacy of letrozole in combination with trastuzumab compared to letrozole monotherapy as first-line treatment in patients with HER2-positive, hormone-receptorpositive metastatic breast cancer - results of the eLEcTRA trial. Breast 2012; 21 (1): 27-33. https://doi.org/10.1016/j. breast.2011.07.006

24. Kaufman B, Mackey JR, Clemens MR, Bapsy PP, Vaid A et al. Trastuzumab plus anastrozole versus anastrozole alone for the treatment of postmenopausal women with human epidermal growth factor receptor 2-positive, hormone receptor-positive metastatic breast cancer: results from the randomized phase III TAnDEM study. J Clin Oncol 2009; 27 (33): 5529-5537. https:// doi.org/10.1200/jco.2008.20.6847
25. Robertson JFR, Steger GG, Neven P, Barni S, Gieseking F et al. Activity of fulvestrant in HER2-overexpressing advanced breast cancer. Annals of Oncology 2010; 21 (6): 1246-1253. https://doi.org/10.1093/annonc/mdp447

26. Tolaney SM, Wardley AM, Zambelli S, Hilton JF, TrosoSandoval TA et al. Abemaciclib plus trastuzumab with or without fulvestrant versus trastuzumab plus standard-of-care chemotherapy in women with hormone receptor-positive, HER2-positive advanced breast cancer (monarcHER): a randomised, open-label, phase 2 trial. Lancet Oncol 2020; 21 (6): 763-775. https://doi.org/10.1016/s1470-2045(20)30112-1 